\title{
A Target Tracking Algorithm based on Fractional Ambiguity Function in Impulsive Noise Environment
}

\author{
Xiaofei Shi ${ }^{1, a}, \mathrm{Li} \mathrm{Li}^{2}$ \\ ${ }^{1}$ Information Science and Technology College, Dalian Maritime University, Dalian, 116026, China \\ 2 Information Engineering College, Dalian University, Dalian, 116622, China
}

\begin{abstract}
This paper proposes an airplane tracking algorithm based on study of the problem of interference localization. Firstly, a novel signal model to accurately estimate parameters of the airplane is proposed in impulsive noise environment. A method of instantaneous Doppler frequency estimation based on peak searching of the fractional lower-order ambiguity function based on the fractional Fourier transform (FLOS_FAF) is proposed, and a method of projection approximation subspace tracking using robust m-estimation method based on fractional lowerorder ambiguity function in fractional Fourier transform domain (FF-RLM_PAST) is proposed to estimate the azimuth angle and elevation angle. As a result, the airplane tracking is achieved in bistatic radar, laying the foundations for interference localization. The correctness and effectiveness of the proposed method are verified with the computer simulation.
\end{abstract}

\section{Introduction}

With increase of radio stations quantity, the interfered probability of civil aviation radio frequency increases. It is key point how to make it safe operation of civil aviation radio frequency. Interference localization is realized by utilizing Doppler frequency shifts of the scattered signals from civil airplane in the receiver ${ }^{[1],[2]}$. If real-time state information of airplane is not obtained, interference localization is not realized. It is shown that accurate estimation of airplane state information is very important.

Studies and experimental measurements have shown that a class of $\alpha$-stable distributions is more appropriate for modeling impulsive noise than Gaussian distribution in signal processing applications ${ }^{[3]}$. Since the stable distribution does not have finite second-order moments ( $1 \leq \alpha<2)$, or even first-order moment $(\alpha<1)$ due to the heavy tails, the performance of the existing parameter estimation and target tracking methods based on secondorder will degrade severely.

In research background of the problem of interference localization, this paper addresses the problem of parameter estimation and target tracking in the presence of impulsive noise. With combination of the fractional ambiguity function with the fractional lower order statistics, a parameter estimation and airplane tracking algorithm is presented in this paper.

\section{The Proposed Signal Model}

In this paper, the airplane tracking and localization is realized by employing the principle of parameter estimation in bistatic Multiple-Input Multiple-Output radar system. Fig.1 illustrates a bistatic MIMO radar system. The considered bistatic MIMO radar is composed of $M$ transmit antennas and $N$ receive antennas with an interelement spacing of $\lambda / 2 . \mathrm{D}$ is the base line distance between the transmit reference element and the receive reference element. $\theta_{\mathrm{t}}(t), \varphi_{\mathrm{t}}(t)$ and $\theta_{\mathrm{r}}(t), \varphi_{\mathrm{r}}(t)$ are azimuth angle and elevation angle correspond to the transmit array and receive array, respectively. The transmitting antennas emit orthogonal waveforms $x_{m}(t)$ for $m=1, \ldots, M$. The received signals contain timevariant Doppler due to the three dimension motion of airplane. If the cubic phase is ignored, the performance of parameter estimation and airplane tracking will degrade. This paper presents a new signal model for the bistatic MIMO radar system. The echo of the $n$th receiving antenna is

$$
\begin{aligned}
s_{n}(t)= & \sum_{m=1}^{M} x_{m}(t) \exp \left(j 2 \pi\left(\eta t+\mu t^{2}+\kappa t^{3}\right)\right) . \\
& a_{t m}\left(\theta_{t}, \varphi_{t}\right) a_{r n}\left(\theta_{r}, \varphi_{r}\right)+w_{n}(t)
\end{aligned}
$$

where $\eta, \mu$, and $\kappa$ are Doppler frequency parameters. $a_{t m}\left(\theta_{t}, \varphi_{t}\right)=\exp \left(-j \pi(m-1) \sin \theta_{t}(t) \cos \varphi_{t}(t)\right)$ is the $\mathrm{mth}$ element of the transmitter steering vector, $a_{r n}\left(\theta_{r}, \varphi_{r}\right)=\exp \left(-j(\pi(n-1)+2 \pi D / \lambda) \sin \theta_{r}(\mathrm{t}) \cos \varphi_{r}(\mathrm{t})\right)$ is the steering vector of the nth element of the receiver.

\footnotetext{
$\overline{{ }^{a} \text { Corresponding author: Shi_xiao_fei_57@163.com }}$
} 
The noise $w_{n}(t)$ is a sequence of i.i.d isotropic complex $S \alpha S$ random variable with $1<\alpha \leq 2$.

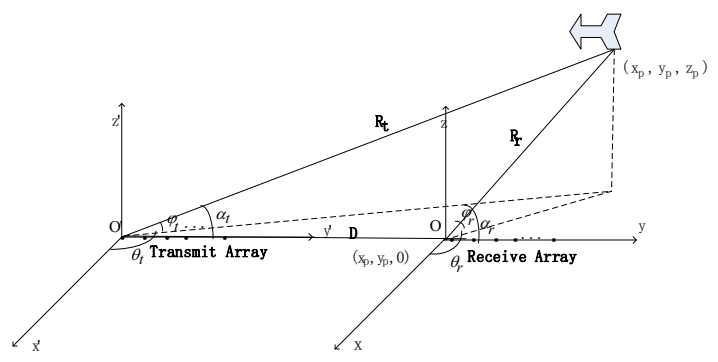

Figure 1. Bistatic MIMO radar system

Since the transmitted waves are orthogonal with each other and orthonormal, there exist two conditions, such as $\left\langle x_{q}, x_{k}\right\rangle=0, q \neq k$ and $\left\|x_{q}\right\|^{2}=1$, for $k=1, \ldots, Q$ and $\boldsymbol{A}_{t}(\theta, \varphi)=\left[a_{t 1}(\theta, \varphi), \ldots, a_{t M}(\theta, \varphi)\right]^{\mathrm{T}} q=1, \ldots, Q$. At each receiving antenna, these orthogonal waveforms can be extracted by $Q$ matched filters. The output of the mth matched filter at the $n$th receive antenna can be expressed as

$$
s_{m n}(t)=D(\eta, \mu, \kappa) a_{t m}\left(\theta_{t}, \varphi_{t}\right) a_{r n}\left(\theta_{r}, \varphi_{r}\right)+w_{n}(t)
$$

Where $D(\eta, \mu, \kappa)=\exp \left(j 2 \pi\left(\eta t+\mu t^{2}+\kappa t^{3}\right)\right)$. The vector of all output of $M$ matched filter can be expressed as

$$
\boldsymbol{S}(t)=\boldsymbol{A} D(\eta, \mu, \kappa)+w_{n}(t)
$$

where $\boldsymbol{A}=\boldsymbol{A}_{t}(\theta, \varphi) \otimes \boldsymbol{A}_{r}(\theta, \varphi), \otimes$ is Kronecker product, and $\boldsymbol{A}_{r}(\theta, \varphi)=\left[a_{r 1}(\theta, \varphi), \ldots, a_{r N}(\theta, \varphi)\right]$.

\section{Fractional ambiguity function based on fractional lower-order statistics}

The ambiguity function is a useful tool for describing the ability of a waveform to simultaneously estimate the range and range-rate (speed) of targets in active (correlation-based) radar and sonar systems [4]. In recent years, a new time-frequency analysis tool, the Fractional Fourier transform, attracts increasingly more attention in signal processing and is widely applied to detection, parameter estimation and direction-of-arrival estimation of the linear frequency modulation signal.

With the development of the fractional Fourier transform (FRFT), an important relationship between the FRFT representation of the narrowband ambiguity function and its transform under the rotation operator is studied. In [5], a new ambiguity function theory based on the FRFT is presented to solve the problem of parameter estimation.

\subsection{Fractional Ambiguity Function}

Assume that the cubic phase signal $r(t)$ is modeled as

$$
r(t)=b_{0} \exp \left(j 2 \pi\left(a_{0}+a_{1} t+a_{2} t^{2}+a_{3} t^{3}\right)\right)+w(t)
$$

where $b_{0}$ is the signal amplitude, and $a_{i}, i=0,1,2,3$ are signal phase factors, the amplitude and phase factors are real and unknown . Instantaneous autocorrelation function $R_{r}(t, \tau)$ of the signal $r(t)$ is defined by

$$
\begin{aligned}
R_{r}(t, \tau) & =r\left(t+\frac{\tau}{2}\right) r^{*}\left(t-\frac{\tau}{2}\right)+R_{w}(t, \tau) \\
& =b_{0}^{2} \exp \left(j 2 \pi\left(3 a_{3} \tau t^{2}+2 a_{2} \tau t+a_{1} \tau+a_{3} \tau^{3} / 4\right)\right) \\
& +R_{w}(t, \tau)
\end{aligned}
$$

where $\tau$ denote delay, $R_{w}(t, \tau)$ is treated as random interference.

For a given delay $\tau, R_{r}(t, \tau)$ has the characteristics of linear frequency modulation signal, which contains noise. According to the definition of FRFT in [6], fractional ambiguity function (FAF) of the signal $r(t)$, namely the FRFT of (5), $F A F_{s}(\rho, m, \tau)$ can be written as

$$
F A F_{r}(\rho, m, \tau)=\int_{-\infty}^{+\infty} R_{r}(t, \tau) K_{\rho}(t, m) d t
$$

where $\rho$ is the rotation angle and $m$ is the frequency in FRFT domain, $K_{\rho}(t, m)$ can be expressed as

$$
K_{\rho}(t, m)=\left\{\begin{array}{cc}
\mathrm{A}_{\rho} \exp \left(j \pi t^{2} \cot \rho-2 j \pi m t \csc \rho\right) \times \\
\exp \left(j \pi m^{2} \cot \rho\right), & \rho \neq n \pi \\
\delta(t-m), & \rho=2 n \pi \\
\delta(t+m), & \rho=(2 n+1) \pi
\end{array}\right.
$$

where $A_{\rho}=\sqrt{(1-j \cot \rho)}$

According to (6) and (7), we can see that

$$
\begin{aligned}
F A F_{r}(\rho, m, \tau)= & b_{0}^{2} A_{\rho} \exp \left(j \pi\left(m^{2} \cot \rho+2 a_{1} \tau+\frac{a_{3} \tau^{3}}{12}\right)\right) \mathrm{g} \\
& \int_{-T / 2}^{+T / 2} \exp \left(j \pi\left(a_{3} \tau+\cot \rho\right) t^{2}\right) \mathrm{g} \\
& \exp \left(j \pi\left(2 a_{2} \tau-2 m \csc \rho\right)\right) d t+W(\rho, m, \tau)
\end{aligned}
$$

where $W(\rho, m, \tau)$ denotes the FAF of noise. By searching the peak of $F A F_{r}(\rho, m, \tau)$, we can obtain the following expressions

$$
\left.\begin{array}{l}
\left(\rho_{0}, m_{0}\right)=\underset{\rho, m}{\arg \max }\left[F A F_{r}(\rho, m, \tau)\right] \\
\operatorname{FAF}_{r}\left(\rho_{0}, m_{0}, \tau\right)=b_{0}^{2} T A_{\rho_{0}} \exp \left(j \pi\left(m^{2} \cot \rho_{0}+2 a_{1} \tau+\frac{a_{3} \tau^{3}}{12}\right)\right) \\
\cot \rho_{0}=-a_{3} \tau \\
m_{0}=a_{2} \tau \sin \rho_{0}
\end{array}\right\}
$$

\subsection{FLOS FAF}

Since the stable distribution does not have finite second-order moments $(1 \leq \alpha<2)$, or even first-order moment $(\alpha<1)$ due to the heavy tails, the performance of the existing signal selective parameters estimation methods based on second-order statistics, such as Instantaneous autocorrelation function, will degrade

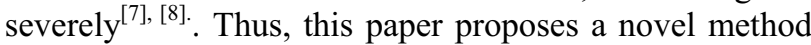
fractional ambiguity function based on fractional lowerorder statistics. 
The fractional lower-order instantaneous autocorrelation function $R_{r}^{(p)}(t, \tau)$ of the signal $r(t)$ is defined by

$$
R_{r}^{(p)}(t, \tau)=\left[r\left(t+\frac{\tau}{2}\right)\right]^{\langle p / 2\rangle}\left[r^{*}\left(t-\frac{\tau}{2}\right)\right]^{\langle p / 2\rangle}
$$

where $p$ is the order of the fractional lower order, and $1<p<\alpha \leq 2$. According to (5) and (6), the FLOS_FAF of the signal $r(t)$ can be written as

$$
F A F_{r}^{(p)}(\rho, m, \tau)=\int_{-\infty}^{+\infty} R_{r}^{(p)}(t, \tau) K_{\rho}(t, m) d t
$$

\section{Parameters Estimation based FLOS_FAF}

\subsection{Doppler Instantaneous Estimation}

According to (9)- (11), the FLOS_FAF of $s_{m n}(t)$ can be written as

$$
F A F_{s, m n}^{(p)}(\rho, m, \tau)=\int_{-\infty}^{+\infty} R_{s, m n}^{(p)}(t, \tau) K_{p}(t, m) d t
$$

Where $R_{s, m n}^{(p)}(t, \tau)=\left[s_{m n}\left(t+\frac{\tau}{2}\right)\right]^{\langle p / 2\rangle}\left[{s_{m n}}^{*}\left(t+\frac{\tau}{2}\right)\right]^{\langle p / 2\rangle}$.

From the relations between $(\rho, m)$ and $\left(a_{2}, a_{3}\right)$, we can get the estimation values of the DFCR and the DFR by finding the peak value of $F A F_{s, m n}^{(p)}(\rho, m, \tau)$

$$
\left.\begin{array}{l}
\hat{\kappa}=-\cot \rho_{0} / 6 \tau \\
\hat{\mu}=m_{0} / 2 \tau \sin \rho_{0}
\end{array}\right\}
$$

Let

$$
y_{1}(t)=s_{m n}(t) \exp \left(-j 2 \pi \hat{\kappa}_{l} t^{3} / 6\right) \cdot \exp \left(-j 2 \pi\left(\hat{\mu}_{l} / 2\right) t^{2}\right)
$$

The fractional autocorrelation function of $y_{1}(t)$ is $R_{y_{1}}^{(p)}(t)=E\left[\left(y_{1}(t)\right)^{\langle p / 2\rangle}\left(y_{1}^{*}(t)\right)^{\langle p / 2\rangle}\right]$. The Fourier transform of $R_{y_{1}}^{(p)}(t)$ is $R_{y_{1}}^{\left(b^{p}\right)}(f)$. Therefore, the IDF $\eta$ can be estimated by

$$
\eta=\arg \max _{f}\left\{R_{y_{1}}^{b}(f)\right\}
$$

\subsection{Azimuth and Elevation Angles Dynamic Recursive Estimation}

Bin Yang [9] proposed the method of projection approximation subspace tracking (PAST) based on least squares estimation, which is sensitive to impulse noise and make the performance of PAST algorithm degraded. On the base of the study of the method of PAST, this paper propose a novel RLM PAST method based on FLOS FCAF (FF_RLM_PAST)

According to (8) and (15), we can define $z(t)$ as

$$
z(t)=\exp \left(j 2 \pi\left(\eta t+\mu t^{2}+\kappa t^{3}\right)\right)
$$

The fractional cross-ambiguity functions $F C A F_{s z, m n}(\rho, m, \tau)$ between $s_{m n}(t)$ and $z(t)$ can be expressed as

$$
F C A F_{s z, m n}(\rho, m, \tau)=\int_{-\infty}^{+\infty} R_{s z, m n}(t, \tau) K_{\rho}(t, m) d t
$$

Where $\quad R_{s z, m n}(t, \tau)=s_{m n}\left(t+\frac{\tau}{2}\right) z^{*}\left(t-\frac{\tau}{2}\right) \quad, \quad W(\rho, m, \tau)$ denotes the FAF of the noise. The $\operatorname{FCAF}_{s z, m n}(\rho, m, \tau)$ arises the peak value when both $\rho$ and $m$ meet the conditions shown in (13). The peak value of the $F C A F_{y z, l}(\rho, m, \tau)$ is

$$
\begin{aligned}
& \operatorname{FCAF}_{s z, m n}\left(\rho_{0}, m_{0}, \tau\right)=T A_{\rho_{0}} a_{t m}\left(\theta_{t}, \varphi_{t}\right) \cdot \\
& a_{r n}\left(\theta_{r}, \varphi_{r}\right) \exp \left(j \pi\left(m^{2} \cot \rho_{0}+2 \eta \tau+\frac{\kappa \tau^{3}}{2}\right)\right)
\end{aligned}
$$

According to (11) to (18), we can obtain the expression of FLOS FCAF as

$$
\begin{array}{r}
F C A F_{s z, m n}^{(p)}\left(\rho_{0}, m_{0}, \tau\right)=a_{t m}\left(\theta_{t}, \varphi_{t}\right) a_{r n}\left(\theta_{r}, \varphi_{r}\right) . \\
F A F_{s z, m n}^{(p)}\left(\rho_{0}, m_{0}, \tau\right)+W^{(p)}\left(\rho_{0}, m_{0}\right)
\end{array}
$$

The output of all receiving antenna based on FLOS FCAF can be expressed as

$$
\boldsymbol{F C} \overline{\boldsymbol{A}} \boldsymbol{F}=\left[\mathrm{FCAF}_{s z, 11}^{(p)}, \ldots, \mathrm{FCAF}_{s z, 1 N}^{(p)} ; \ldots ; \ldots \mathrm{FCAF}_{s z, M N}^{(p)}\right]
$$

Both receive subarrays $\boldsymbol{R}_{1}$ and $\boldsymbol{R}_{2}$ constructed in this paper can be expressed by

$$
\begin{aligned}
& \boldsymbol{R}_{1}=\left[\begin{array}{lll}
\boldsymbol{F C} \boldsymbol{A} \boldsymbol{F}_{11} & \mathrm{~L} & \boldsymbol{F C} \boldsymbol{A} \boldsymbol{F}_{1 N}
\end{array}\right]=\boldsymbol{A}_{r}(\theta, \varphi) D+\boldsymbol{N}_{1} \\
& \boldsymbol{R}_{2}=\left[\begin{array}{lll}
\boldsymbol{F} \boldsymbol{C} \boldsymbol{A} \boldsymbol{F}_{11} & \mathrm{~L} & \boldsymbol{F C} \boldsymbol{A} \boldsymbol{F}_{M 1}
\end{array}\right]^{\mathrm{T}}=\boldsymbol{A}_{\boldsymbol{t}}(\theta, \varphi) D+\boldsymbol{N}_{2}
\end{aligned}
$$

Next, the proposed method of FF_RLM_PAST is introduced in detail by taking the subarray $\boldsymbol{R}_{1}$ as an example.

The matrix $\boldsymbol{W}(t)$ denotes the signal subspace of observation vector $\boldsymbol{R}_{1}$. This signal subspace is estimated by the minimization of a cost function $J_{\text {FF_RLM_PAST }}(\boldsymbol{W}(t))$ in the FF_RLM_PAST algorithm.

$$
J_{\text {FF_RLM_PAST }}(\boldsymbol{W}(t))=\sum_{n=1}^{t} \beta^{t-n} \rho\left(\left\|\boldsymbol{R}_{1}(n)-\boldsymbol{W}(t) \boldsymbol{y}(n)\right\|_{2}^{2}\right)
$$

where $0<\beta<1$ denotes the forgetting factor. $\boldsymbol{y}(n)=\boldsymbol{W}^{H}(t-1) \boldsymbol{R}_{1}(n)$ is projection approximation vector. $\rho(\cdot)$ is M-estimation function, whose derivative is denoted as $\varphi(\cdot)=\rho^{\prime}(\cdot)$. Reference [11] indicated that evaluation function of Cauchy distribution function can obtain good preference in alpha-stable distribution noise with different characteristic exponent when parameter estimation is studied by employing M-estimation method in alpha-stable distribution noise.

We expect $J_{\text {FF_RLM_PAST }}{ }^{\prime}(\boldsymbol{W}(t))$ to be a good approximation for $J_{\text {FF_RLM_PAST }}(\boldsymbol{W}(t))$ and the matrix $\boldsymbol{W}(t)$ minimizing $J_{\text {FF_RLM_PAST }}{ }^{\prime}(\boldsymbol{W}(t))$ to be a good estimate for the signal subspace of $\boldsymbol{C}(t)$. $J_{\text {FF_RLM_PAST }_{-}}(\boldsymbol{W}(t))$ is minimized if

$$
\boldsymbol{W}(t)=\boldsymbol{C}_{\boldsymbol{R}_{1} \boldsymbol{y}}(t) \boldsymbol{C}_{\boldsymbol{y} y}^{-1}(t)
$$




$$
\begin{aligned}
& \boldsymbol{C}_{\boldsymbol{R}_{1} \boldsymbol{y}}(t)=\sum_{n=1}^{t} \beta^{t-n} \varphi\left(\|\boldsymbol{e}(n)\|_{2}^{2}\right) \boldsymbol{R}_{1}(n) \boldsymbol{y}^{\boldsymbol{H}}(n) \\
& \boldsymbol{C}_{\boldsymbol{y} \boldsymbol{y}}(t)=\sum_{n=1}^{t} \beta^{t-n} \varphi\left(\|\boldsymbol{e}(n)\|_{2}^{2}\right) \boldsymbol{y}(n) \boldsymbol{y}^{\boldsymbol{H}}(n) \\
& \boldsymbol{e}(t)=\boldsymbol{R}_{1}(t)-\boldsymbol{W}(t-1) \boldsymbol{y}(t)
\end{aligned}
$$

We use RLS algorithm for updating $\boldsymbol{W}(t)$ without derivations. We apply $\boldsymbol{W}(t)$ to the spatial spectrum of 2D-MUSIC algorithm,

$$
P\left(\theta_{\mathrm{r}}, \varphi_{\mathrm{r}}\right)=\frac{1}{\boldsymbol{A}_{r}^{\mathrm{H}}\left(\theta_{\mathrm{r}}, \varphi_{\mathrm{r}}\right) \boldsymbol{W} \boldsymbol{W}^{\mathrm{H}} \boldsymbol{A}_{\boldsymbol{r}}\left(\theta_{\mathrm{r}}, \varphi_{\mathrm{r}}\right)}
$$

Similarly, the algorithm of FF_RLM_PAST is applied to observation vector $\boldsymbol{R}_{2}$. We can obtain as

$$
P\left(\theta_{\mathrm{t}}, \varphi_{\mathrm{t}}\right)=\frac{1}{\boldsymbol{A}_{t}^{\mathrm{H}}\left(\theta_{\mathrm{t}}, \varphi_{\mathrm{t}}\right) \boldsymbol{W} \boldsymbol{W}^{\mathrm{H}} \boldsymbol{A}_{t}\left(\theta_{\mathrm{t}}, \varphi_{\mathrm{t}}\right)}
$$

Searching spectral peak of $P\left(\theta_{\mathrm{r}}, \varphi_{\mathrm{r}}\right)$ and $P\left(\theta_{\mathrm{t}}, \varphi_{\mathrm{t}}\right)$, we can realize the azimuth angle $\theta_{\mathrm{r}}(t), \theta_{\mathrm{t}}(t)$ and elevation angle $\varphi_{\mathrm{r}}(t), \varphi_{\mathrm{t}}(t)$ dynamical recursive estimation.

\section{Simulation results}

The considered bistatic MIMO radar is composed of $M=6$ transmit antennas and $N=8$ receive antennas with an interelement spacing of $0.5 \mathrm{~m}$. The base line distance between the transmit reference element and the receive reference element is $D=5 \mathrm{Km}$. Since the alphastable process has infinite variance for $\alpha<2$, we use a generalized signal-to-noise ratio (GSNR) measure defined as the ratio of the signal power over the implusive noise dispersion $\gamma$, $\operatorname{GSNR}=10 \log _{10}\left(\mathrm{E}\left\{|r(\mathrm{t})|^{2}\right\} / \gamma\right)$. According to this choice of GSNR metric, the $S \alpha S$ noise samples are power scaled by the dispersion parameter $\gamma$. The number of Monte Carlo iterations is 500 in all simulations.

\section{Simulation 1: Time series $t$}

In this simulation, the noise is modeled as the $\alpha$ stable noise with $\alpha=1.4$, GSNR $=12 \mathrm{~dB}$. Fig. 2 plots the estimation performance of Doppler. From Fig.2, we can find the curve of estimated Doppler is more close to the curve of true Doppler. Therefore, the proposed method has good estimation performance about three Doppler parameters.

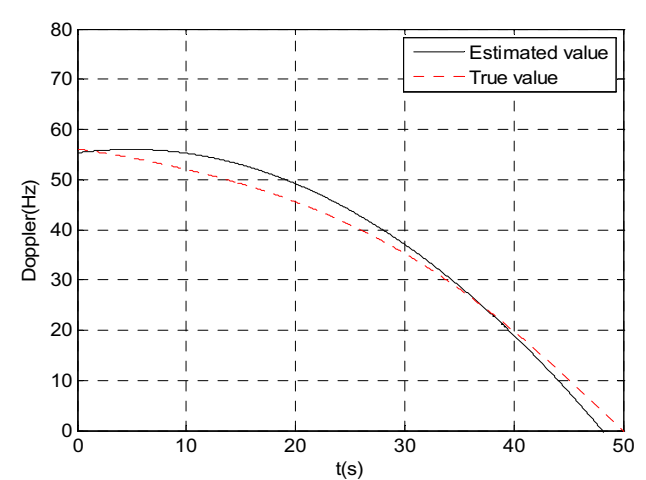

Figure 2. Estimation performance of Doppler

Fig. 3 show curves of azimuth angle and elevation angle dynamical recursive estimators with time $t$. From Fig.3, we can find the curves of estimated azimuth angle and elevation angle are more close to the curves of true value. From Fig.2 and Fig.3, we can find that the proposed method has good estimation performance.

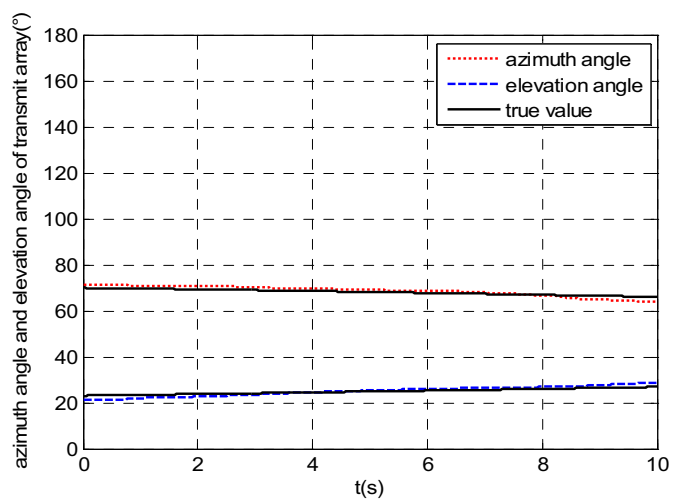

(a)

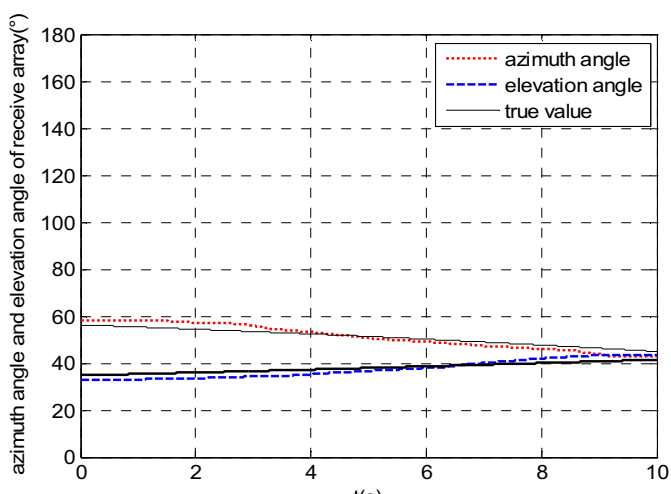

(b)

Figure 3. estimation performance of angle and airplane location

Simulation 2: Characteristic exponent $\alpha$.

In this simulation, the generalized signal to noise ratio is set as $\mathrm{GSNR}=12 \mathrm{~dB}$. Let root mean-squared errors (RMSE) based on average is defined by $\operatorname{RMSE} 2=\frac{1}{N_{s}} \sqrt{\sum_{t=1}^{t=N_{s}}(\hat{\xi}(t)-\xi(t))^{2}}$, where $\hat{\xi}$ is estimator.

Fig. 4 plots the RMSE of the proposed method versus various values of characteristic exponent $\alpha$. The smaller the characteristic exponent $\alpha$ is, the stronger the pulse characteristic is. From Fig.4, we can find that the RMSE of the parameters decreases with the increase of the 
characteristic exponent $\alpha$. Therefore, the proposed method can suppress the interference of impulse noise, and has good performance. From these figures, we also find that the estimation performance of the Doppler frequency parameters affects the estimation performances of the azimuth angle and elevation angle.

\section{Simulation 3: Generalized signal-noise-ratio GSNR}

In this simulation, the noise is modeled as $\alpha$-stable noise with $\alpha=1.4$. Fig.5 show the RMSE of angle and Doppler frequency for various values of the GSNR . From Fig.5, we can find that the proposed method still has better performance in the condition of the lower SNR.

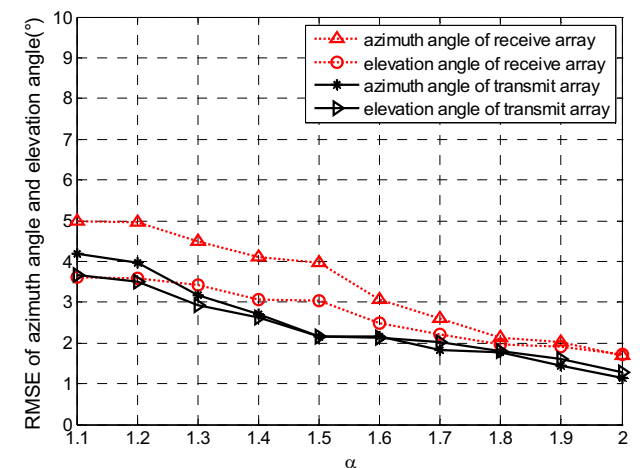

(a)

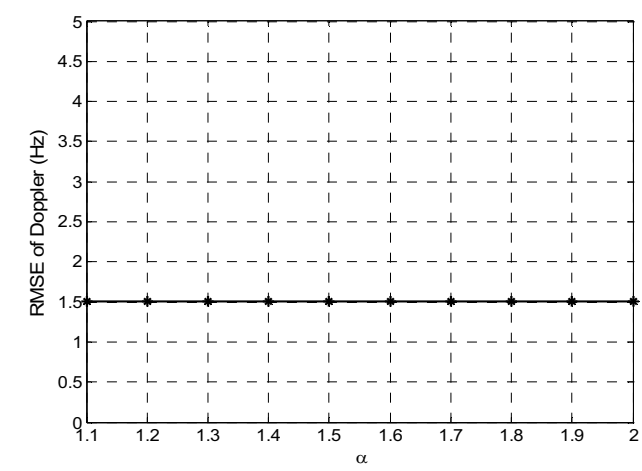

(b)

Figure 4. Root mean square error as a function of the characteristic exponent $\alpha$

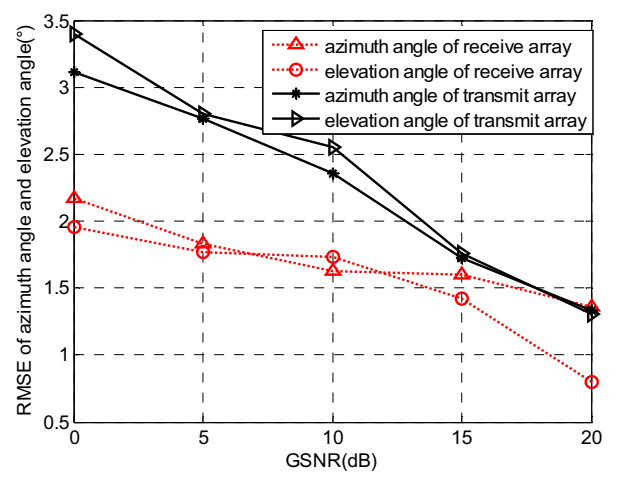

(a)

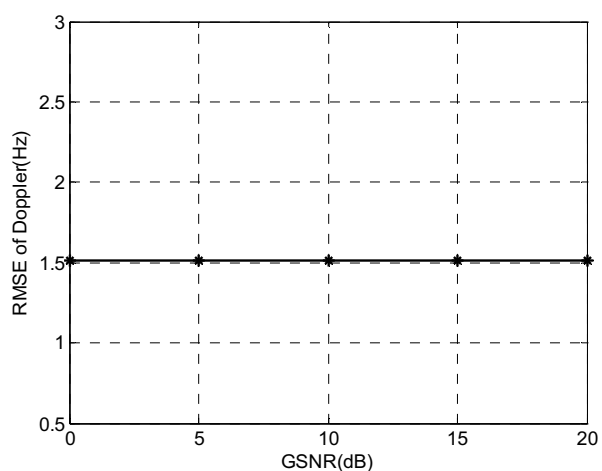

(b)

Figure 5. Root mean square error as a function of the GSNR

\section{Conclusion}

This paper proposes an airplane tracking algorithm based on study of the problem of interference localization. Firstly, a novel signal model is proposed in impulsive noise environment. A method of instantaneous Doppler frequency estimation based on peak searching of the FLOS FAF is proposed, and FF-RLM PAST algorithm is proposed to dynamical recursive estimate the azimuth angle and elevation angle. As a result, the airplane tracking is achieved in bistatic radar, laying the foundations for interference localization. The correctness and effectiveness of the proposed method are verified with the computer simulation.

\section{Acknowledgments}

This work was partly supported by the National Science Foundation of China under Grants 61401055 and the fund of Key Laboratory of marine management technology in State Oceanic Administration People's Republic of China under Grant 201504.

\section{References}

1. H. Tan, J. Li, "Emitter locating algorithm by scattered signals of civil airplanes," Radio communications Technology, vol. 35, pp. 40-42, Feb 2009.(in Chinese)

2. T.S. Qiu, N. Xia, J.C. Li, “An interference localization algorithm based on Gaussian approximation particle filtering with stable distribution noise," Signal Processing, vol. 28, pp. 1248-1253, Sep 2012. (in Chinese)

3. X. Li, W.D. Jiang, Z. W. Zhuang, "MIMO radar sensitivity analysis of antenna position for direction finding," IEEE Transaction on Signal Processing, vol. 60, pp. 5201-5216, Oct 2012.

4. A.A. Gorji, R. Tharmarasa, T. Kirubarajan, "Widely Separated MIMO versus Multistatic Radars for Target Localization and Tracking," IEEE 
Transactions on Aerospace and Electronic Systems, vol.49, pp:2179-2194, April 2013.

5. Q. Qu, M.L. Jin, J.M. KIM, "FRFT based parameter Estimation of the Quadratic FM signal," Chinese Journal of Electronics, vol.19, pp: 463-467, Mar 2010.

6. S.H. Liu, S. Tao, R. Tao, Y.D. Zhang, G. Zhang; F. Zhang, Y. Wang, "Sparse Discrete Fractional Fourier Transform and Its Applications," IEEE Transactions on Signal Processing, vol. 62, pp: 6582 - 6595, Dec 2014.

7. J. Park, G. Shevlyakov, K. Kim, "Maximin distributed detection in the presence of impulsive alpha-stable noise," IEEE Transactions on Wireless Communications, vol. 10, pp.1687-1691, June 2011.

8. C.L. Nikias, M. Shao, "Signal Processing with Alpha-stable Distribution and Application. New York: John Wiley \& Sons, Inc, 1995.

9. Y. Bin, "Projection approximation subspace tracking," IEEE Transaction on Signal Processing, vol. 43, pp. 95-107, Jan 1995.

10. S. Li, T.S. Qiu, "A robust past algorithm in alpha stable noise environment," Acta Electronica Sinica, vol.37, pp. 519-522, Mar 2009. 